\title{
Philippe Martin (dir.), Ephemera catholiques. L'imprimé au service de la religion $\left(\mathrm{XVI}^{\mathrm{e}}-\mathrm{XXI}^{\mathrm{e}}\right.$ siècles)
}

Paris, Beauchesne, coll. « Bibliothèque Beauchesne ", 2012, 383 p.

Jean-Bruno Renard

(2) OpenEdition

Journals

Édition électronique

URL : http://journals.openedition.org/assr/24655

DOI : $10.4000 /$ assr.24655

ISSN : $1777-5825$

Éditeur

Éditions de l'EHESS

Édition imprimée

Date de publication : 30 décembre 2012

Pagination : 237

ISSN : 0335-5985

Référence électronique

Jean-Bruno Renard, «Philippe Martin (dir.), Ephemera catholiques. L'imprimé au service de la religion (XV|e-XX|e siècles) ", Archives de sciences sociales des religions [En ligne], 160 | octobre-décembre 2012, mis en ligne le 19 mars 2013, consulté le 21 septembre 2020. URL : http://journals.openedition.org/ assr/24655; DOI : https://doi.org/10.4000/assr.24655

Ce document a été généré automatiquement le 21 septembre 2020.

(c) Archives de sciences sociales des religions 


\section{Philippe Martin (dir.), Ephemera catholiques. L'imprimé au service de la religion $\left(\mathrm{XVI}^{\mathrm{e}}-\mathrm{XXI}^{\mathrm{e}}\right.$ siècles)}

Paris, Beauchesne, coll. « Bibliothèque Beauchesne ", 2012, 383 p.

Jean-Bruno Renard

\section{RÉFÉRENCE}

Philippe Martin (dir.), Ephemera catholiques. L'imprimé au service de la religion (XVI ${ }^{\mathrm{e}}$ XXI ${ }^{e}$ siècles), Paris, Beauchesne, coll. «Bibliothèque Beauchesne », 2012, 383 p. 
1 Professeur des universités en histoire moderne, spécialiste du religieux, Philippe Martin a notamment déjà publié une somme sur les livres de piété en France (Une religion des livres, 1640-1850, Paris, Le Cerf, 2003, 622 p., compte rendu ASSR 128.32). Il s'intéresse ici à une forme très répandue, quoique paradoxalement fort peu étudiée, d'imprimés, les ephemera, terme recouvrant des productions très diverses qui, dans le domaine religieux, vont des faire-part de mariage ou de décès aux tracts militants, en passant par des feuilles de prière et des bulletins paroissiaux. L'ouvrage se compose de quinze contributions qui méritent d'être détaillées.

Le premier texte, dû à Philippe Martin («Premières approches des ephemera religieux », p.9-61), est une introduction

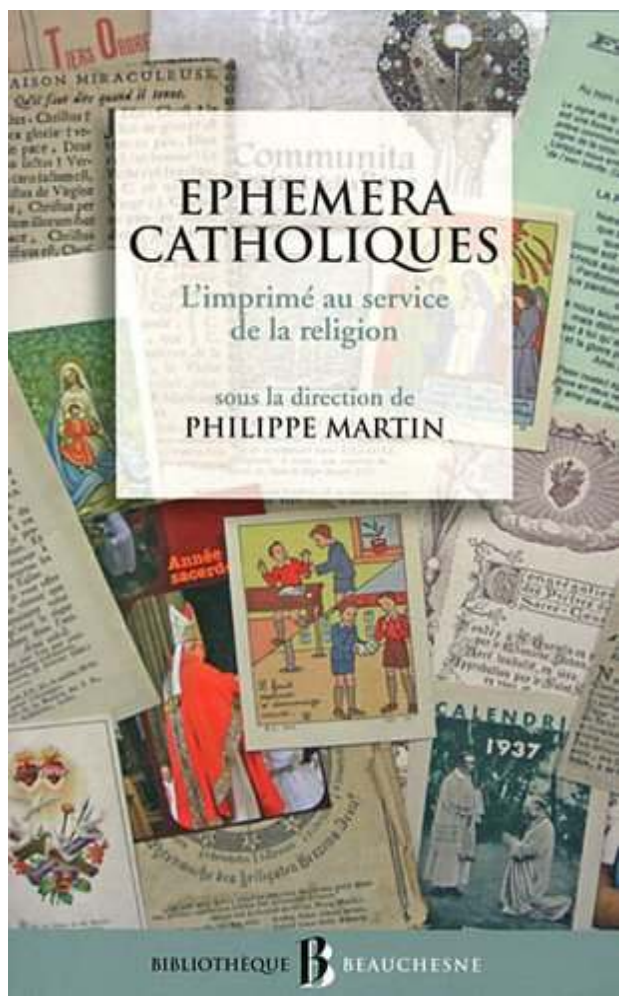
générale particulièrement utile qui constitue un véritable petit essai sur le phénomène des ephemera. Dès la fin du XIX ${ }^{\mathrm{e}}$ siècle, des chercheurs se sont intéressés à ces imprimés souvent laissés pour compte au profit des livres et dont la conservation n'est due qu'à des amateurs et des collectionneurs: tracts, libelles, faire-part, prospectus, programmes, menus, cartes de visite, invitations, etc. Ces imprimés ont été désignés sous le nom de "feuilles volantes ", de "vieux papiers ", de " littérature de rue » et, depuis 1962, d'ephemera, afin de souligner leur caractère éphémère et marginal. Les ephemera religieux remontent à la naissance de l'imprimerie : Philippe Martin rappelle que le premier texte imprimé par des caractères mobiles selon le procédé de Gutenberg, au milieu du $\mathrm{Xv}^{\mathrm{e}}$ siècle, a été non pas la Bible mais des lettres d'indulgences, formulaires sur lesquels on ajoutait à la main le nom du bénéficiaire. Les ephemera religieux peuvent être classés en quatre domaines. Ce sont d'abord les actes religio-administratifs tels que les indulgences, les souvenirs de communion, les attestations de pèlerinage, les certificats de baptême ou d'ordination, les faire-part de cérémonies religieuses (baptême, mariage, décès). Ce sont ensuite les actes de mobilisation idéologique: libelles et pamphlets (ils ont joué un rôle important lors des querelles religieuses, protestants/catholiques ou jansénistes/jésuites), tracts de courants religieux (catholiques progressistes, catholiques traditionalistes), appels au denier du culte... En troisième lieu, des ephemera proposent des actes de dévotion en diffusant des « occasionnels " sur des miracles, des prières adressées à des saints, des brochures en faveur de la béatification de telle ou telle personnalité. Enfin, il y a des actes de pastorale avec les feuilles distribuées pour la célébration de la messe du dimanche, les bulletins paroissiaux, les livrets de catéchisme, etc. On notera que ces imprimés éphémères sont l'équivalent d'actes, destinés à fournir ou à obtenir du croyant des attestations de sa foi. Cette typologie peut se combiner puisqu'un document comme une brochure en faveur d'une béatification, par exemple, relève à la 
fois d'un acte de dévotion et d'un acte de mobilisation. Philippe Martin nous convainc ainsi que «le monde des ephemera est [...] d'une infinie richesse, reflet de toutes les spiritualités et de tous les combats » (p. 58). C'est dire combien l'exploitation de ce type de documents peut être précieuse pour l'historien comme pour le sociologue, voire pour le psychologue des religions.

3 La première partie de l'ouvrage rassemble des textes qui traitent de la question de la collecte, de la conservation et du classement des ephemera religieux. Historien et collectionneur, Dominique Lech, "Quelques réflexions sur une collection d'ephemera " (p.65-90) invite à exploiter les archives des imprimeurs qui rendent compte des «travaux de ville ", c'est-à-dire l'impression d'ephemera par opposition à l'impression d'ouvrages. Sa collection de plus d'un millier de pièces, imprimées entre 1671 et aujourd'hui, témoigne majoritairement, selon l'auteur, d'une pédagogie de la peur fondée sur un catholicisme intransigeant. Elisabetta Gulli-Grigioni ("Annonces et souvenirs mortuaires italiens », p. 91-95) montre la «culture de la mort» (p. 92) que reflètent ces faire-part de décès. En découvrant les feuillets de souvenirs mortuaires que son grand-père avait placés dans un livre de piété - quatre feuillets pour des camarades tombés au front et un feuillet pour les parents de son aïeul-, Catherine Martin, "Une Imitation de Jésus Christ "farcie" " (p. 97-117) voit dans cette pratique chargée d'émotion un "petit monument aux morts personnel»(p.100). Cécile de Cacqueray et Pierre Moracchini, bibliothécaires à la Bibliothèque Franciscaine des Capucins de Paris, présentent le riche fonds d'ephemera franciscains conservés dans cet établissement et évoquent les problèmes de collecte, de conservation, de datation, de droit d'auteur, de normes descriptives pour ce type d'imprimés («Les ephemera en bibliothèque ", p. 119-133).

La deuxième partie de l'ouvrage décline la diversité de la pastorale catholique à travers les ephemera des siècles passés. Bruno Maes ("Pèlerinages et ephemera miraculeux ", p. 137-158) étudie les feuillets imprimés relatant un miracle : ceux du $\mathrm{XvI}^{\mathrm{e}}$ siècle sont encore tout imprégnés de merveilleux médiéval, tandis que ceux du $\mathrm{xVIII}^{\mathrm{e}}$, sous l'influence du rationalisme religieux, ont pour rôle de prouver la vérité d'une doctrine. Éric Suire, «Autour du culte des saints (XVII ${ }^{\mathrm{e}}$-XVIII ${ }^{\mathrm{e}}$ siècles)»(p. 159-177), s'intéresse aux mandements épiscopaux diffusés dans les paroisses pour annoncer une béatification, ou instituer une fête de saint, ou réprimer des abus, ainsi qu'aux biographies simplifiées de saints et de bienheureux. Ces documents prouvent que le culte des saints ne relève pas de la seule religion populaire, mais est régulé par l'autorité ecclésiastique. Julien Léonard, «Récits de conversion de juifs à Metz au XVII siècle » (p.179-205) analyse un corpus original dont le faible nombre (quatre ephemera) est inversement proportionnel à la publicité faite à l'époque à ces conversions et aux cérémonies de baptême qui les couronnent. Les récits, assez stéréotypés, avec des motifs récurrents comme l'action du Saint-Esprit, les tentatives de récupération du converti par la communauté juive et la protection accordée par le clergé, n'étaient pas destinés aux Juifs mais aux catholiques et, indirectement, dirigés contre les Réformés. Jean-Marc Lejuste, "Ephemera et vies exemplaires de Visitandines» (p. 207-239) exploite un corpus du XVIII ${ }^{\mathrm{e}}$ siècle de notices nécrologiques de sœurs visitandines du couvent de Metz rédigées par la Mère supérieure et envoyées aux autres monastères de l'ordre. Ces textes, «abrégés de la vie et des vertus » (p. 210), sont de précieux témoignages sur l'origine sociale des Visitandines, le mécanisme de leur conversion et les qualités morales que l'on attendait d'elles. La contribution d'Alain J. Lemaître ( Les placards 
mortuaires au XVIII ${ }^{e}$ siècle ", p. 241-257) souligne le rôle de distinction sociale rempli par ces annonces de funérailles à Rennes dans les milieux de noblesse de robe, ainsi que l'évolution de la sensibilité mortuaire tout au long du siècle, depuis l'image macabre et tragique du «De profundis » jusqu'à l'apaisement préromantique du « Requiescat in pace ». Fabienne Henryot étudie un corpus d'ephemera produits par un pensionnat catholique de Nancy dans la seconde moitié du XIXe siècle ( $"$ Les ephemera au temps de la lutte pour la liberté de l'enseignement, La Malgrange, 1836-1901», p. 259-287), exploitant des documents aussi variés que les publicités pour l'établissement, les feuillets d'information sur la vie scolaire, les brochures relatives aux fêtes organisées par les élèves du collège et enfin les discours de distribution des prix. Au Québec à la même époque, dans les ephemera de l'enseignement catholique, c'est moins la défense de l'enseignement privé contre l'enseignement public qui se manifeste que la concurrence entre établissements catholiques, ainsi que le montre Christine Hudon dans sa contribution « Des prospectus pour promouvoir les collèges catholiques pour garçons au Québec (xIx -xxe siècle)»(p. 289-305). Enfin Philippe Martin («L'âge d'or des ephemera ", p. 307-332) étudie les ephemera catholiques militants de la première moitié du $\mathrm{xx}^{\mathrm{e}}$ siècle : sur le modèle des tracts de propagande communiste et socialiste, et contre eux, ces ephemera vont être largement utilisés par les mouvements d'un «catholicisme d'action» (p.332) qui fleurit durant cette période au travers des "Croisades» (Croisade mariale, Croisade de la Messe, Croisade du Rosaire) et du scoutisme catholique.

5 La troisième et dernière partie de l'ouvrage présente deux contributions concernant le temps présent. Le premier texte («Une communication d'Église à Nancy durant le carême 2010 », p. 335-367) présente les résultats d'un travail réalisé par un groupe d'étudiants de Master 2 d'histoire religieuse de l'Université de Nancy (Charlotte Dessaint, Alban Didillon, Sébastien Dupont, Manuel Manhard, Julie Pierrat, Typhanie Ravanello et Damien Vitry). Ils ont systématiquement collecté les feuillets et brochures disponibles sur des tables ou des présentoirs dans les églises de Nancy en février-mars 2010, soit cent cinquante-deux documents différents. La diffusion est très locale : cinq ephemera étaient présents dans plus de quatre églises contre cent vingt qui n'étaient présents que dans une seule église. Qu'ils soient de fabrication artisanale ou professionnelle, ces ephemera couvrent la variété des activités de la vie catholique aujourd'hui : textes para-liturgiques (chants pour la messe, prières, textes de réflexion spirituelle), vie de groupes (activités spécifiques pour les enfants, les jeunes couples, les retraités, les scouts), appels de fonds (denier du culte, Secours catholique - suite au tremblement de terre en Haïti en janvier 2010), informations (annonces paroissiales, cycles de conférences, tract pour des bibliothèques religieuses, organisation de retraites spirituelles et de pèlerinages). Les auteurs montrent le rôle joué par les prêtres de la paroisse dans la sélection des documents mis à la disposition des fidèles. Raison de plus pour regretter l'absence dans cet ouvrage d'une contribution sur ces feuillets photocopiés déposés sur des bancs d'église ou au pied de statues, expression d'une religiosité superstitieuse faite de prières aux saints des causes désespérées (sainte Rita, saint Expédit ou saint Jude) et prenant parfois la forme de "chaines magiques ». Le dernier texte déroge, mais avec bonheur, à la délimitation de l'ouvrage au domaine catholique en traitant des ephemera jéhovistes : Jean-Pierre Chantin (« Les Témoins de Jéhovah, de tracts en brochures ", p. 369-383) montre que ces imprimés sont inséparables de l'activité de porte-à-porte du prosélytisme des Témoins et que leur 
production aux États-Unis à des fins de diffusion mondiale leur donne un caractère universel gommant les spécificités nationales. 